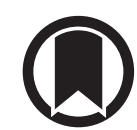

CrossMark

\title{
Declining trend in incidence of tuberculosis in adolescents and young adults in Taiwan
}

\author{
To the Editor:
}

SNow et al. [1] presented the results of a global estimate of the incidence of tuberculosis (TB) in adolescents and young adults. Owing to the incomplete age disaggregation and underestimated reports, the authors used case detection rates and age structure of the population in different countries to infer the total number of adolescents and young adults who developed TB in 2012. The results showed that young people had a considerable burden of TB, accounting for $17 \%$ of all new TB cases. However, the accuracy of the study is probably limited owing to the uncertainty of some key parameters.

Here, we provide valuable epidemiological reports of TB in Taiwan, a TB-endemic area with an intermediate TB burden. For all newly diagnosed active TB cases, irrespective of sputum smear status and including both pulmonary and extrapulmonary TB cases, notification of the Taiwan Centers for Disease Control (CDC) is mandatory and a surveillance system was well established. In this system, TB cases were disaggregated into 5-year age groups. The directly observed treatment short-course (DOTS) programme implementation and active contact investigation started in 2006.The TB incidence rate in Taiwan was 72.5 cases per 100000 population in 2005 and 43.9 cases per 100000 population in 2016. To clarify the TB burden of adolescents and young adults in Taiwan, we analysed the trends of notified TB patients aged 10-24 years from 2005 to 2016. The cases were identified based on the registration database of the Taiwan $\mathrm{CDC}$, in which cases can be diagnosed by bacteriological or pathological evidence, or clinically diagnosed due to good response to anti-TB treatment.

During the study period, 8160 adolescent and young adult TB cases were identified, accounting for 5.23\% of all cases (8160/156033). As shown in figure 1, the annual number of these TB cases in young people decreased in total by $61.73 \%$, from 1006 in 2005 to 385 in 2016 . The proportion of these young people also decreased by $2.3 \%$, from $6.11 \%$ in 2005 to $3.81 \%$ in 2016 . On the contrary, during the study period, the number of patients aged $\geqslant 65$ years decreased in total by $31.01 \%$ (from 8408 in 2005 to 5801 in 2016) and increased in proportion by $5.13 \%$ (from $51.04 \%$ to $56.17 \%$ ). In patients aged $25-64$ years, the number of cases declined by $41.20 \%$ (from 6997 in 2005 to 4114 in 2016) and decreased in proportion by $2.65 \%$ (from $42.48 \%$ in 2005 to $39.83 \%$ in 2016 ). The younger population apparently had a faster decline trend in TB incidence than the older population (figure 1). We then compared the proportions of male and female TB cases in different age aggregations from 2005 to 2016. The sex ratio was generally equal in the patients aged 10-30 years (figure 1). After 30 years of age, the proportion of male cases increased and that of female cases declined gradually. In the patients aged $>65$ years, the proportion of male to female cases was approximately $75 \%$ to $25 \%$.

In our nationwide analysis, we found that the TB burden of the young population in Taiwan is lower than that reported by SNOw et al. [1]. Moreover, we observed a clear trend of better TB control and a faster decline in annual incidence in the young population compared to the older population. To improve TB control, Taiwan initiated the Half TB Action Plan since 2005, which integrated active case finding, the directly observed treatment short-course (DOTS) programme, aggressive contact investigation and improved treatment for multidrug-resistant TB. The estimated number of household contacts per TB case

@ERSpublications

The incidence of tuberculosis in adolescents and young adults in Taiwan has a faster declining trend than that in the elderly population after implementation of the directly observed treatment shortcourse programme and active contact investigation http://ow.ly/X8hN30ok9PJ

Cite this article as: Chen Y-Y, Pan S-W, Shen H-S, et al. Declining trend in incidence of tuberculosis in adolescents and young adults in Taiwan. Eur Respir J 2019; 53: 1801305 [https://doi.org/10.1183/ 13993003.01305-2018]. 


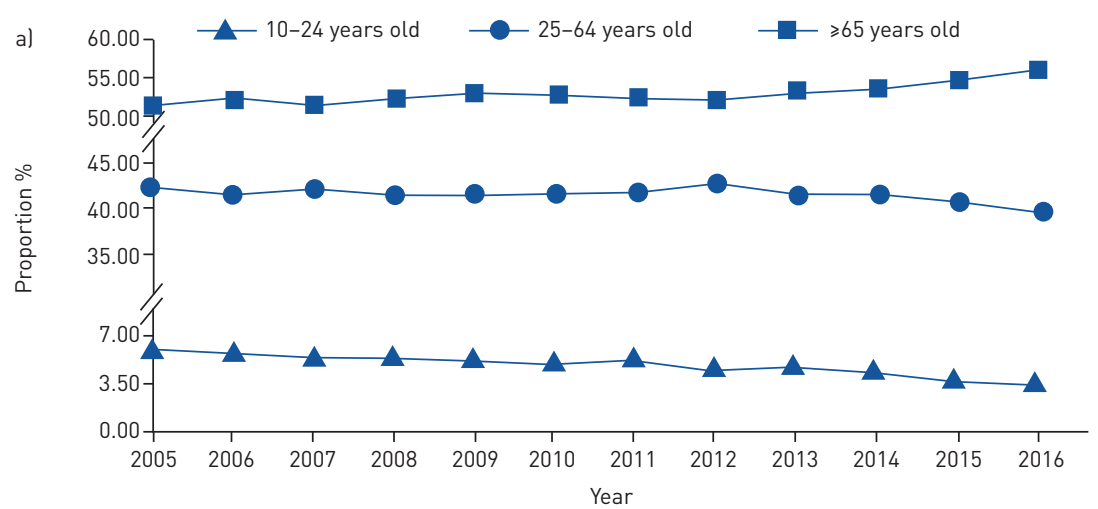

\begin{tabular}{|c|c|c|c|c|c|c|c|c|c|c|c|c|}
\hline Age & 2005 & 2006 & 2007 & 2008 & 2009 & 2010 & 2011 & 2012 & 2013 & 2014 & 2015 & 2016 \\
\hline \multicolumn{13}{|l|}{$\geqslant 65$ years } \\
\hline Number & 8408 & 8029 & 7477 & 7451 & 7078 & 7000 & 6609 & 6424 & 6112 & 6054 & 5856 & 5801 \\
\hline Change compared with $2005 \%$ & & -4.51 & -11.07 & -11.38 & -15.82 & -16.75 & -21.40 & -23.60 & -27.31 & -28.00 & -30.35 & -31.01 \\
\hline \multicolumn{13}{|l|}{ 25-64 years } \\
\hline Number & 6997 & 6383 & 6112 & 5949 & 5476 & 5524 & 5300 & 5293 & 4804 & 4733 & 4386 & 4114 \\
\hline Change compared with $2005 \%$ & & -8.78 & -12.65 & -14.98 & -21.74 & -21.05 & -24.25 & -24.35 & -31.34 & -32.36 & -37.32 & -41.20 \\
\hline \multicolumn{13}{|l|}{$10-24$ years } \\
\hline Number & 1006 & 908 & 830 & 816 & 731 & 672 & 696 & 587 & 581 & 515 & 433 & 385 \\
\hline Change compared with $2005 \%$ & & -9.74 & -17.50 & -18.89 & -27.34 & -33.20 & -30.82 & -41.65 & -42.25 & -48.81 & -56.96 & -61.73 \\
\hline
\end{tabular}

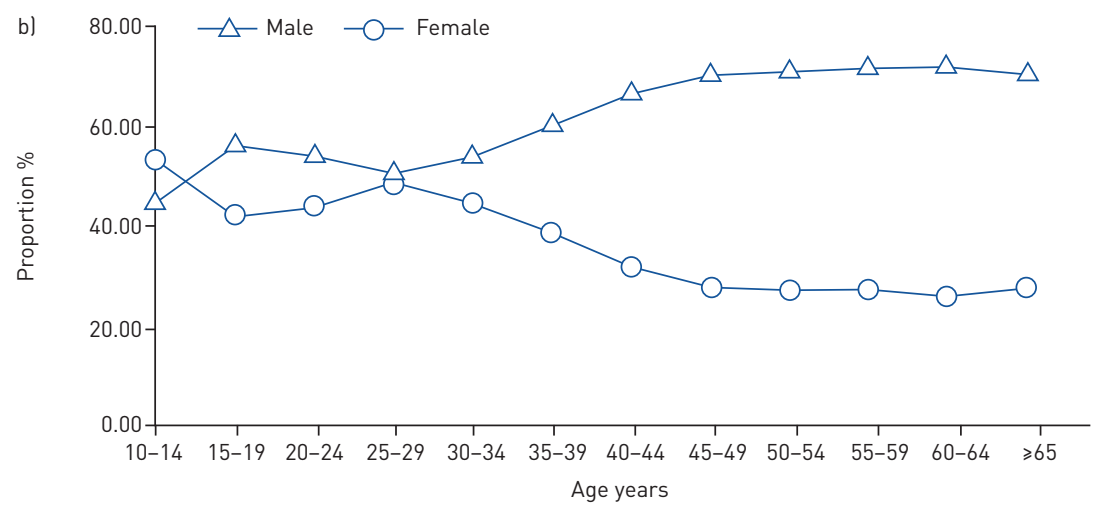

FIGURE 1 Proportions of notified tuberculosis cases among various age groups and sexes in Taiwan from 2005 to 2016. a) Patients with tuberculosis are categorised into ages $10-24,25-64$, and $\geqslant 65$ years, and their annual changes in proportions are presented. The percentage changes of the number of cases in each year in comparison with the number of cases in 2005 (baseline) are also shown. b) The proportions of male and female patients with tuberculosis by 5 -year age aggregation.

rose from 2.2 in 2006 up to 12.0 in 2017. The proportion of TB contacts that received chest radiography was $88 \%$ in 2006 and was $96 \%$ in 2017 . The notified TB cases continued to decrease after 2006, which indicated that aggressive contact investigation did not determine an increase in TB incidence rate in Taiwan. Meanwhile, several previous studies demonstrated that DOTS improved treatment outcomes [2], reduced acquired second-line drug-resistant TB and multidrug-resistant TB [3, 4], and reduced TB-specific mortality in TB patients in Taiwan [5]. Previous studies reported that young patients acquired TB primarily from recent household or community transmission [6, 7], and may have compromised treatment adherence in some areas [8]. Therefore, adolescents and young adults with TB clearly benefit more from the DOTS programme and active contact investigation. On the contrary, older patients usually have active TB due to reactivation from a remote TB infection, and as a result they benefit less from both [9].

Treatment strategies for latent TB infection (LTBI) were initiated LTBI in 2008, but the implementation of LTBI treatment in the whole population only started after 2016. Therefore, preventive LTBI treatment probably only played a minor role in the declined trend of TB incidence observed in the present study.

The sex inequality in TB incidence has been a well-known phenomenon worldwide for decades [10]. However, the sex disparities among patients with TB of various ages have rarely been evaluated. In this analysis, we found an obvious sex disparity in patients with TB who were aged $>35$ years. However, the sex ratio in young patients with $\mathrm{TB}$ aged $<30$ years was generally equal. The coverage rate of national health 
insurance in Taiwan is $>99 \%$. Therefore, the difference in medical accessibility between the sexes would be a minor issue [11].

Other known factors which have been commonly discussed include smoking, alcoholism, diabetes mellitus and HIV infection. In Taiwan, the increasing trend of smoking and alcohol use disorder in elderly men $[12,13]$ should be considered as an element in sex disparities. The higher prevalence of diabetes in women than in men [14] indicated that diabetes is less likely to be a cause of sex inequality.

The prevalence of HIV/AIDS in Taiwan is low: $0.04 \%$ in 2005 and $0.12 \%$ in 2016 . The rate of HIV/TB co-infection increased from $0.5 \%$ in 2001 to $3.1 \%$ in 2016 among newly diagnosed TB patients aged from 15 to 49 years old, and it was much higher in men than in women. The peak incidence of HIV/TB co-infection occurred at age 35-39 years old and declined gradually. Considering the relative low proportion of HIV/TB co-infection and inconsistent sex distribution profile between general TB patients and those with HIV/TB co-infection, we believe that the contribution of HIV/TB co-infection to sex disparity is relatively low in Taiwan. The differences in socioeconomic status, such as income level and living conditions, should also be considered, but cannot be clearly evaluated in the present study.

There are several limitations in our study. The registration database relating to the time period before 2005 is incomplete and the DOTS status of each individuals was not available, therefore the effect of the DOTS programme cannot be clearly investigated. Several important clinical, bacteriological, and socioeconomical characteristics of the notified cases are not available in the database, therefore these important confounders cannot be corrected in our analysis. It also limited our abilities to identify the key components that contributed to the different declining trends in various age populations.

In summary, our nationwide analysis depicted the declining trend in $\mathrm{TB}$ in adolescent and young adult patients in Taiwan. The remarkable decline in $\mathrm{TB}$ incidence in the young population highlights the importance of the DOTS programme and active contact investigation in TB control. Meanwhile, we observed different sex disparities between the younger and older populations. Further investigation of the socioeconomic issues between the sexes would be helpful to develop age- and sex-specific strategies for TB elimination.

As stated by the World Health Organization, collaboration and fostering strong partnerships between national and international stakeholders, and beyond TB programmes and health sectors, will pave the way to accelerating the elimination of TB in both low- and high-incidence countries [15].

Ying-Ying Chen ${ }^{1}$, Sheng-Wei Pan $\oplus^{1,2,3}$, Hsiang-Shi Shen ${ }^{1}$, Fan-Yi Chuang ${ }^{1}$, Jia-Yih Feng ${ }^{1,2,4,6}$ and Wei-Juin Su ${ }^{2,5,6}$ ${ }^{1}$ Dept of Chest Medicine, Taipei Veterans General Hospital, Taipei, Taiwan. ${ }^{2}$ School of Medicine, National Yang-Ming University, Taipei, Taiwan. ${ }^{3}$ Institute of Public Health, National Yang-Ming University, Taipei, Taiwan. ${ }^{4}$ Institute of Clinical Medicine, National Yang-Ming University, Taipei, Taiwan. ${ }^{5}$ Chest Dept, Taipei Veterans General Hospital, Taipei, Taiwan. ${ }^{6}$ Jia-Yih Feng and Wei-Juin Su contributed equally to this work.

Correspondence: Wei-Juin Su, Dept of Chest Medicine, Taipei Veterans General Hospital, Number 201, Section 2, Shih-Pai Road, Taipei 112, Taiwan. E-mail: wjsu@vghtpe.gov.tw

Received: July 132018 | Accepted after revision: March 012019

Conflict of interest: None declared.

\section{References}

1 Snow KJ, Sismanidis C, Denholm J, et al. The incidence of tuberculosis among adolescents and young adults: a global estimate. Eur Respir J 2018; 51: 1702352.

2 Bloss E, Chan PC, Cheng NW, et al. Increasing directly observed therapy related to improved tuberculosis treatment outcomes in Taiwan. Int J Tuberc Lung Dis 2012; 16: 462-427.

3 Chien JY, Lai CC, Tan CK, et al. Decline in rates of acquired multidrug-resistant tuberculosis after implementation of the directly observed therapy, short course (DOTS) and DOTS-Plus programmes in Taiwan. J Antimicrob Chemother 2013; 68: 1910-1916.

4 Chien JY, Tsou CC, Chien ST, et al. Direct observation therapy with appropriate treatment regimens was associated with a decline in second-line drug-resistant tuberculosis in Taiwan. Eur J Clin Microbiol Infect Dis 2014; 33: 941-948.

5 Yen YF, Yen MY, Lin YP, et al. Directly observed therapy reduces tuberculosis-specific mortality: a population-based follow-up study in Taipei, Taiwan. PLoS One 2013; 8: e79644.

6 Suwanpimolkul G, Jarlsberg LG, Grinsdale JA, et al. Molecular epidemiology of tuberculosis in foreign-born persons living in San Francisco. Am J Respir Crit Care Med 2013; 187: 998-1006.

$7 \quad$ Mathema B, Andrews JR, Cohen T, et al. Drivers of tuberculosis transmission. J Infect Dis 2017; 216: S644-S653.

8 Tesfahuneygn G, Medhin G, Legesse M. Adherence to anti-tuberculosis treatment and treatment outcomes among tuberculosis patients in Alamata district, northeast Ethiopia. BMC Res Notes 2015; 8: 503.

9 Karumbi J, Garner P. Directly observed therapy for treating tuberculosis. Cochrane Database Syst Rev 2015; 5: CD003343.

10 Neyrolles O, Quintana-Murci L. Sexual inequality in tuberculosis. PLoS Med 2009; 6: e1000199. 
11 Wu TY, Majeed A, Kuo KN. An overview of healthcare system in Taiwan. London J Prim Care (Abingdon) 2010; 3: 115-119.

12 Wen CP, Levy DT, Cheng TY, et al. Smoking behaviour in Taiwan, 2001. Tob Control 2005; 14: Suppl. 1, i51-i55.

13 Lee CT, Hsiao CY, Chen YC, et al. Incidence and risk of alcohol use disorders by age, gender and poverty status: a population-based-10 year follow-up study. Neuropsychiatry (London) 2017; 7: 892-896.

14 Jiang YD, Chang CH, Tai TY, et al. Incidence and prevalence rates of diabetes mellitus in Taiwan: analysis of the 2000-2009 nationwide health insurance database. J Forma Med Assoc 2012; 111: 599-604.

15 Lonnroth K, Migliori GB, Abubakar I, et al. Towards tuberculosis elimination: an action framework for low-incidence countries. Eur Respir J 2015; 45: 928-952. 